\title{
Experimental verification of a numerical surface roughness model for metallic bodies under large plastic strain
}

\author{
Pawet Sidun ${ }^{1, *}$ \\ ${ }^{1}$ Faculty of Mechanical Engineering, Bialystok University of Technology, Wiejska 45C, 15-351 Bialystok, Poland
}

\begin{abstract}
The present work describes selected aspects of numerical modelling of the process of bonding metal alloys with consideration for micro-roughness, as well as experimental verification. The plastic-elastic contact between two deformable bodies was investigated in a DEFROM FEM environment, and verified at the test stand. The present paper demonstrates selected results of contact modelling investigated using aluminium-copper samples, in relation to their elastic-plastic range. The real surface profile helped to create a mathematical model of surface roughness measured using a laser microscope. Dependency between a blade of a tool and a feed was used to build a numerical model of roughness based on the arithmetic average value of the roughness profile. The work presents also a process of simplification of real surface roughness for the needs of numerical calculations. The paper investigates an impact of wave roughness at obtained values on effective plastic strain and stress. Additionally, numerical analysis shows a need to enter a new roughness wave correction factor assuming a zero value of the coefficient of friction. This is due to the interaction of metallic surfaces within the plastic contact zone. The obtained results allow the estimation of the impact of surface force interaction expressed by the wave coefficient factor. The experimental verification of numerical calculations allowed the estimation of the actual impact of the micro-cutting process in the entire friction process. Further analysis of obtained results permits the author to explain the surface phenomena occurring during the friction process, such as adhesion or diffusion, and outline the development direction of numerical methods.
\end{abstract}

\section{Introduction}

The development of manufacturing techniques, in particular metal forming, forces engineers to use increasingly sophisticated computer methods aimed at reducing production costs and increasing the geometrical accuracy of finished parts. The finite element method is a tool of modern engineering which is widely used in plastic forming. This method is well suited to the analysis of the plastic flow of metals as well as for the analysis of contact related issues and in regard to elastic and elasticplastic contact with metallic surfaces. Plentiful works addressing the numerical modelling of roughness can be found in literature. Sun and his team [1] investigated the process of flattening sinusoidal surfaces imitating roughness from the perspective of the effect of plastic deformation on wear parameters. Buczkowski and Kleiber dealt with numerical modelling of elastic-plastic surface contact problems connected with nonlinear interface compliance [2]. Broz and Paik's works deal with similar modelling of elastic-plastic surface contact with boundary roughness [4]. It is also possible to find a lot about wear, tribological phenomena and friction models. One such example is the work of Zhang et al., which presents the impact of surface roughness on the effectiveness of the elastic-plastic area of a contact [5]. Dry elastic-plastic contact of nominally flat surfaces was studied by Manoylov et al. [6] while friction analysis of microcosmic elastic-plastic contact for extrusion process was the theme of the work published by Wang et al. [7] An excellent comparison of friction models occurring during metal forming processes was presented by Tan [3]. However, related literature contains no works strictly related to the experimental verification of the numerical model of roughness during friction processes.

The aim of this work is to build a numerical roughness model based on the actual surface model of the tested samples and then verify it experimentally.

\section{Modelling of roughness}

Roughness modelling with relation to real surface profiles using the finite element method (FEM) requires some simplification.

All FEM environments implement these two laws of friction:

Coulomb's law - describes friction within the elastic range:

$$
-\quad \mu=\frac{F_{f}}{N}=\frac{\tau_{f} \cdot A}{\sigma_{n} \cdot A}=\frac{\tau_{f}}{\sigma_{n}} \rightarrow \tau_{f}=\mu \cdot \sigma_{n}
$$

Constant shear stress law - for plastic friction:

$$
-\tau_{f}=f \cdot \bar{\sigma}=m \cdot \frac{\bar{\sigma}}{\sqrt{3}}=m \cdot k
$$

${ }^{*}$ Corresponding author: pawel.sidun.pb@gmail.com 
where: $\tau_{f}$-frictional stress $[\mathrm{MPa}], N$ - normal force $[\mathrm{N}]$, $F_{f}$ - frictional force $[\mathrm{N}], A$ - contact area $\left[\mathrm{mm}^{2}\right]$, $\mu$ - friction coefficient, $m$ - frictional shear factor $0 \leq m \leq 1, k$ - shear yield strength [MPa], $\bar{\sigma}$ - effective stress [MPa], $f$ - friction factor;

The Fourier Series is the simplest, as well as the most common method for describing all kinds of signals, but it also turns out to be useful in describing the roughness model.

For integer $N \geq 1$ the Fourier series is expressed as:

$$
-\quad S_{N}=\frac{A_{0}}{2}+\sum_{i-1}^{N} A_{i} \sin \left(\frac{2 \pi i x}{T}+\varphi_{i}\right)
$$

For $i=1, A_{0}=0, \varphi_{1}=0, T=2 \pi$ equation (3) takes the form of equation (4):

$$
-\quad S_{N}=A_{1} \sin (x)
$$

Equation 4 is used to describe the geometry of surfaces after the turning process. Fig. 1 presents the process of simplifying a real surface profile into a character numerical model.

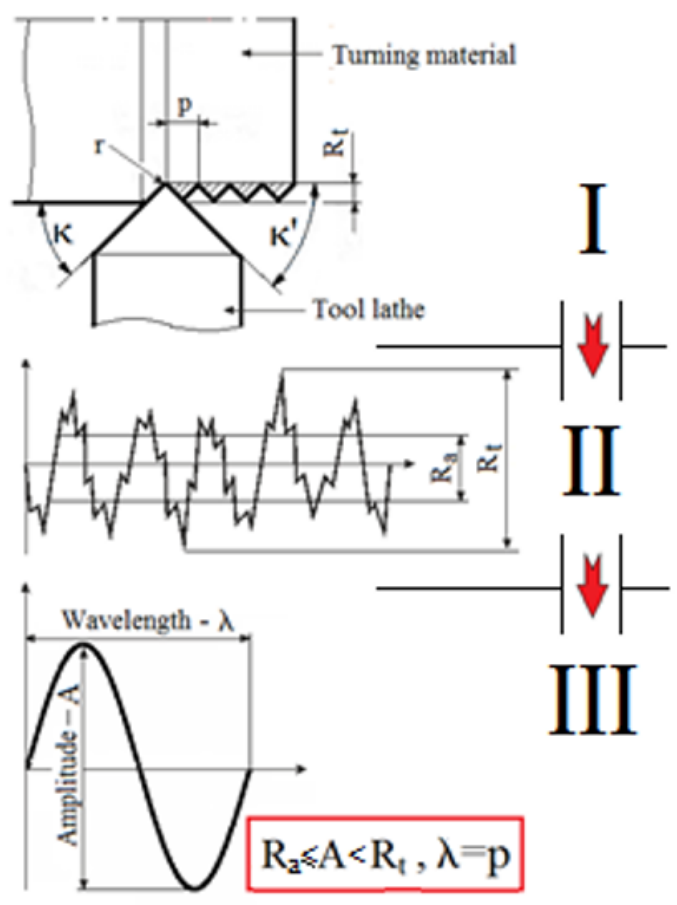

Fig. 1. Stages of modelling physical roughness, I - method of surface formation through the turning process, II - real surface profile obtained through measurement, III - simplification of the roughness model.

$$
\begin{gathered}
-R_{t}=p \frac{\tan (\kappa) \cdot \tan \left(\kappa^{\prime}\right)}{\tan (\kappa)+\tan \left(\kappa^{\prime}\right)} \\
-\quad R_{a}=\frac{p^{2}}{32 r}
\end{gathered}
$$

where: $R_{t}$-theoretical maximum roughness $[\mathrm{mm}], R_{a^{-}}$arithmetic average values of the roughness profile, $p$ - feed $[\mathrm{mm} / \mathrm{rev}], \kappa$-primary and secondary lead angle of the lathe $\left[{ }^{\circ}\right], r$-radius of the corner.
Literature provides some dependencies equation 5 and 6 that are commonly known in machining problems, describing the relations between the received roughness value and the feed rate in relation to the turning process. Thanks to those relationships, as well as roughness values obtained using a 3D LEXT OLS4000 laser microscope, results obtained from the measurements along with appropriate input data from the turning process can be systematised, making it possible to construct a numerical model based on the roughness parameter $R_{a}$ and the feed rate $p$. Parameters $R_{a}=A=$ $10 \mu \mathrm{m}$ and $\lambda=p=50 \mu \mathrm{m}$ were used to build a model for an alloy of $\mathrm{Cu}-\mathrm{ETP}$ copper and AW-6060 aluminium.

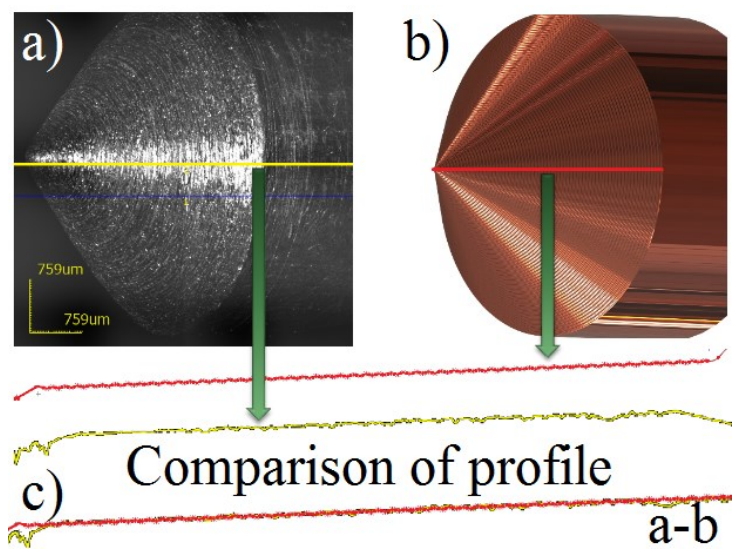

Fig. 2. A comparison of the real surface profile and the numerical model, a) real surface profile, b) numerical model, c) comparison of wave roughness $a-b$.

The presented comparison of both roughness profiles Fig. 2. visually shows a high geometrical compatibility, whereas the difference between the numerical model and the experimental test can be seen through the numerical analysis of the bonding process.

\section{FEM analysis of the bonding process}

The current work presents the experimental verification of numerical roughness modelling depicted in Fig. 3.

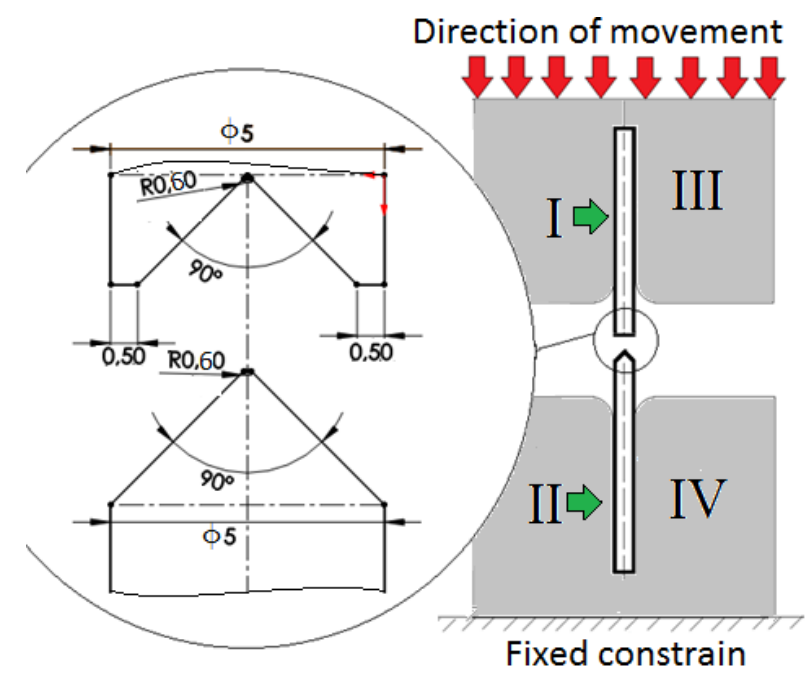

Fig. 3. Schematic of the issue in question. 
The numerical analysis of the elastic-plastic bond was carried out in the DEFORM 2D computational environment on $\phi 5 \mathrm{~mm}$ diameter samples made of $\mathrm{Cu}$ ETP and AW-6060 Fig. 4.

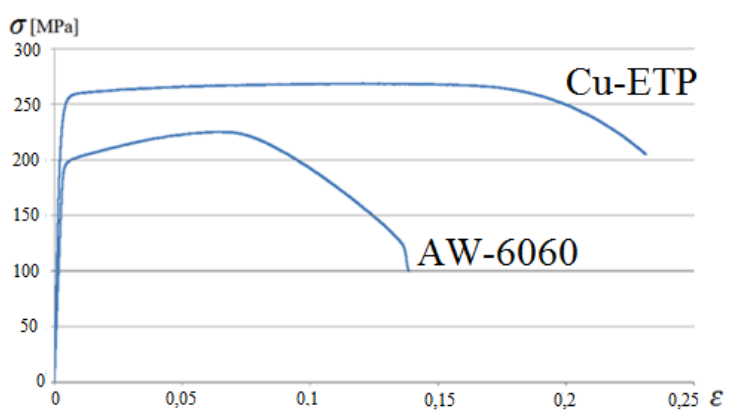

Fig. 4. Stress-strain curves of both materials at constant strain rate equal $0,02 s^{-1}$ and temperature $20^{\circ} \mathrm{C}$ of tensile test.

The elastic-plastic model is based on the $\mathrm{HMH}$ criterion, as well as on the model of isotropic material hardening. In the experiment, as well as in the calculations, it has been assumed that the speed of the upper die remains constant and be equal to a value of 0.02 $\mathrm{mm} / \mathrm{s}$ Fig. 3. - III, as well as that the temperature remains at $20^{\circ} \mathrm{C}$. Rigid elements - III and IV, elastic-plastic - I and II, the friction between elements III - I and IV - II have been described using a friction coefficient of 0.08 obtained through utilisation of graphite grease. For construction of the numerical model 15,000 quad elements, presented in Fig. 5, were used for each specimen.

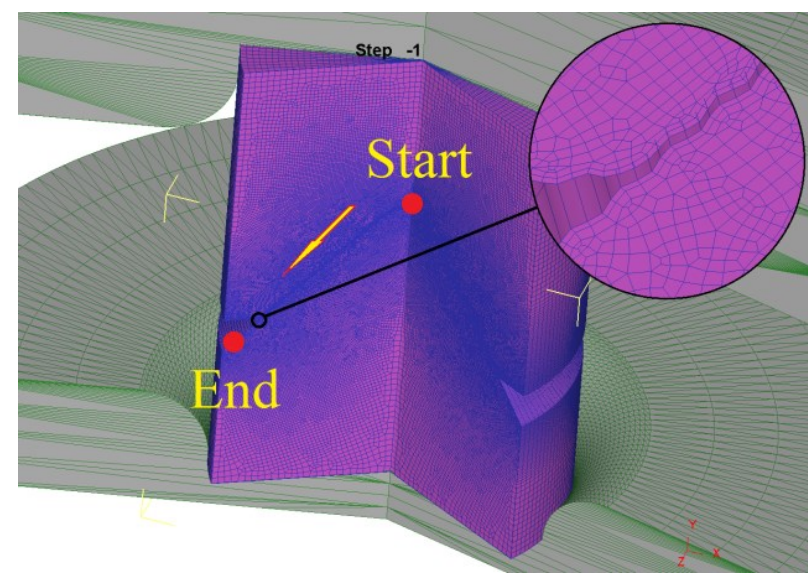

Fig. 5. A 3D representation of a 2D Numerical model of the issue with the indication of the direction of measurement along the surfaces.

\section{Verification of the numerical model}

The experimental verification of the numerical roughness model showed that it is necessary to apply a correction factor for a model with a roughness wave. Results of the experiments show a high geometrical compatibility of the tested profiles with the assumption of a roughness wave and the correction coefficient

Fig. 6.

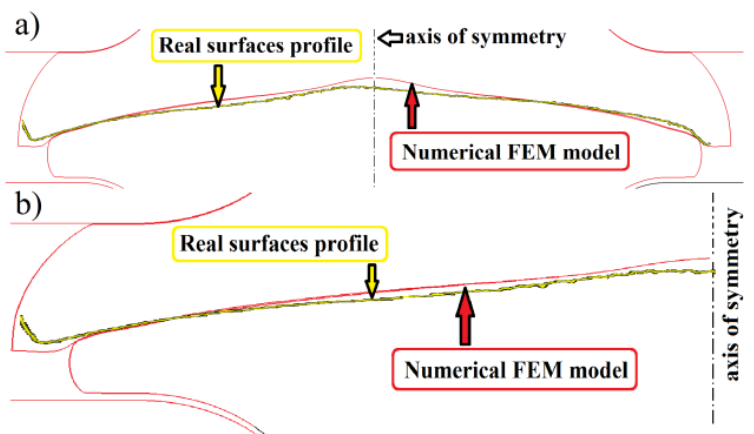

Fig. 6. Experimental verification of a numerical model after spring-back, a) entire surface profile b) half profile.

Correction coefficient for the pair of samples in contact zone I and II was determined on the basis of experimental verification:

$$
-w=\frac{\sum v}{2} \cdot \frac{\sum R_{e}}{\sum R_{m}}
$$

where: $v$-Poisson's ratio of materials, $R_{e}$ - Yield's strength of materials [MPa], $R_{m}$ - Ultimate strength of materials $[\mathrm{MPa}]$.

The obtained factor is treated by the computing environment as a friction factor equation 7 but it already fulfils a different role in the presented issue. The effect of the micro-cutting process was estimated using a sinusoidal roughness wave model built using FEM mesh while the surface force interaction was estimated using a "wave parameter" " $\boldsymbol{w}$ ". The "wave parameter" coefficient accounts for Poisson's ratio, which results from the flattening of the roughness in the contact zone causing the bearing capacity of the surface in the subsequent stages of deformation to increase. The increase in the load capacity at the electron level, on the other hand, causes an increase in electrostatic interactions between particular particles and a consequence of the cohesive force at the level of macromolecules. In addition, the coefficient considers the influence of material parameters such as $\mathrm{Re}$ and $\mathrm{Rm}$ affecting the speed of contact surface deformation causing a reduction in the distance between the two real surfaces, i.e. an increase in load capacity. It should also be remembered that the tested factor is valid only for the finite element method.

\section{Effect of micro-roughness}

Numerical analysis of the bonding process shows the effect of micro-cutting within the entire friction process. In the first phase of deformation Fig. 7 with the force of $4.5 \mathrm{kN}$, the influence of micro geometry is very significant as can be seen in the diagram. The maximum value of effective strain equal 1.82 (In DEFORM environment results of effective strain is multiplied 10times) and marked on Fig. 7. This point is very important for deformation process because at surface generates a small curvature that ultimately confers the geometry shape of the sample, according with experimental results. In subsequent stages of deformation, the effect of micro geometry decreases, and surface forces become more significant (Fig. 8.) due to the flattening of the roughness wave and its almost complete disappearance. 

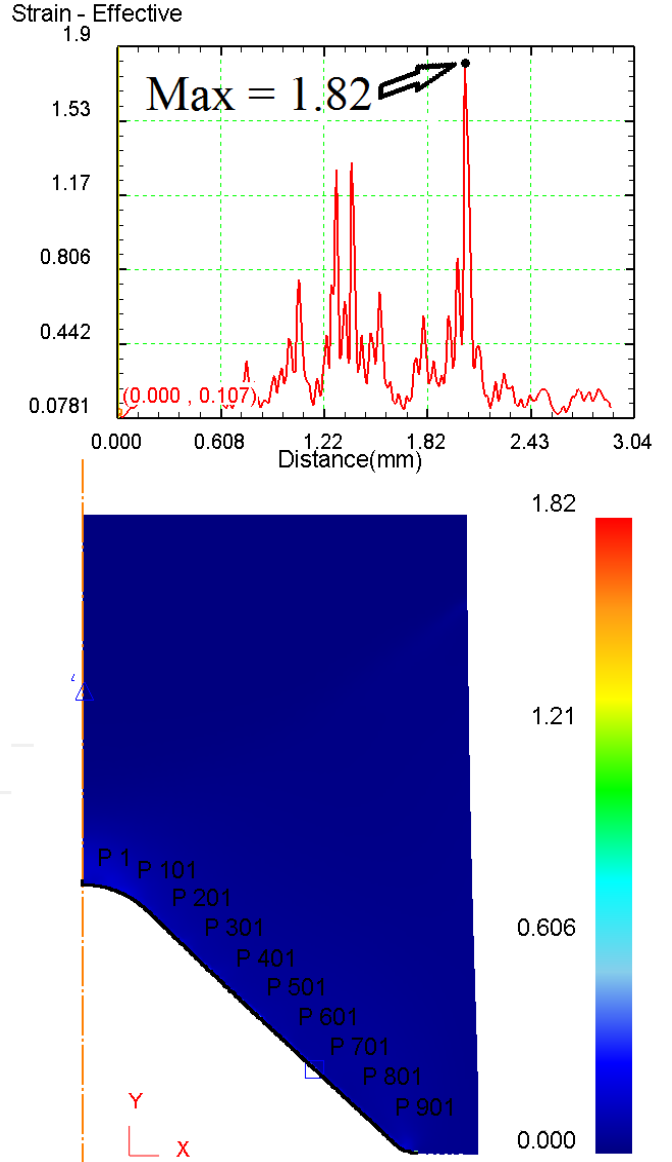

1.82

Fig. 7. Initial stage of deformation, axial force equal to $4.5 \mathrm{kN}$.
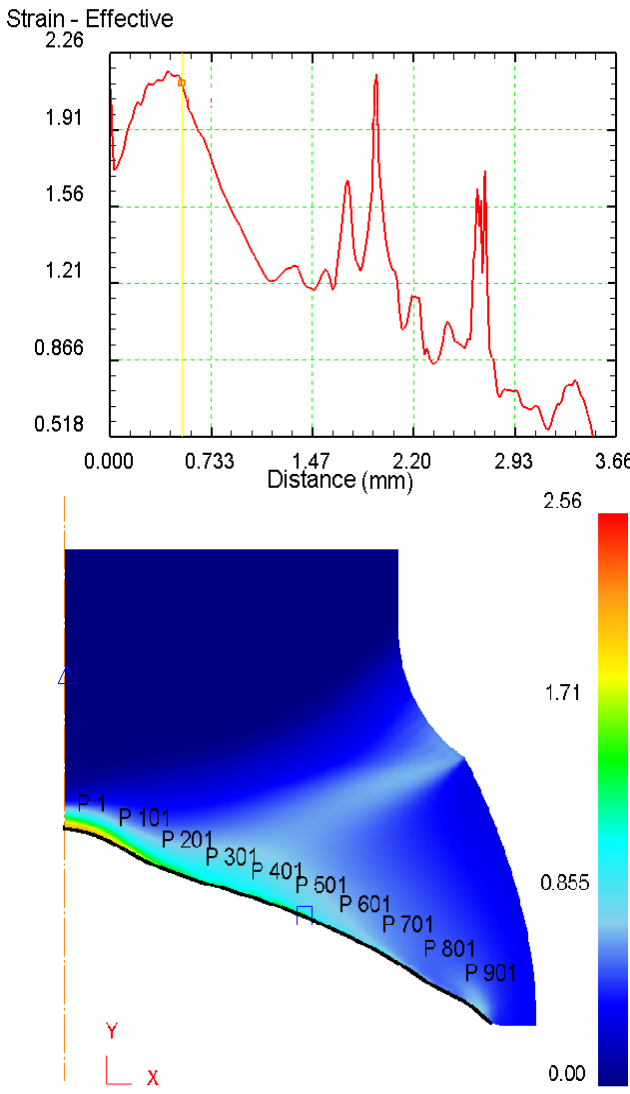

Fig. 8. Subsequent stage of deformation, axial force equal to $10 \mathrm{kN}$.

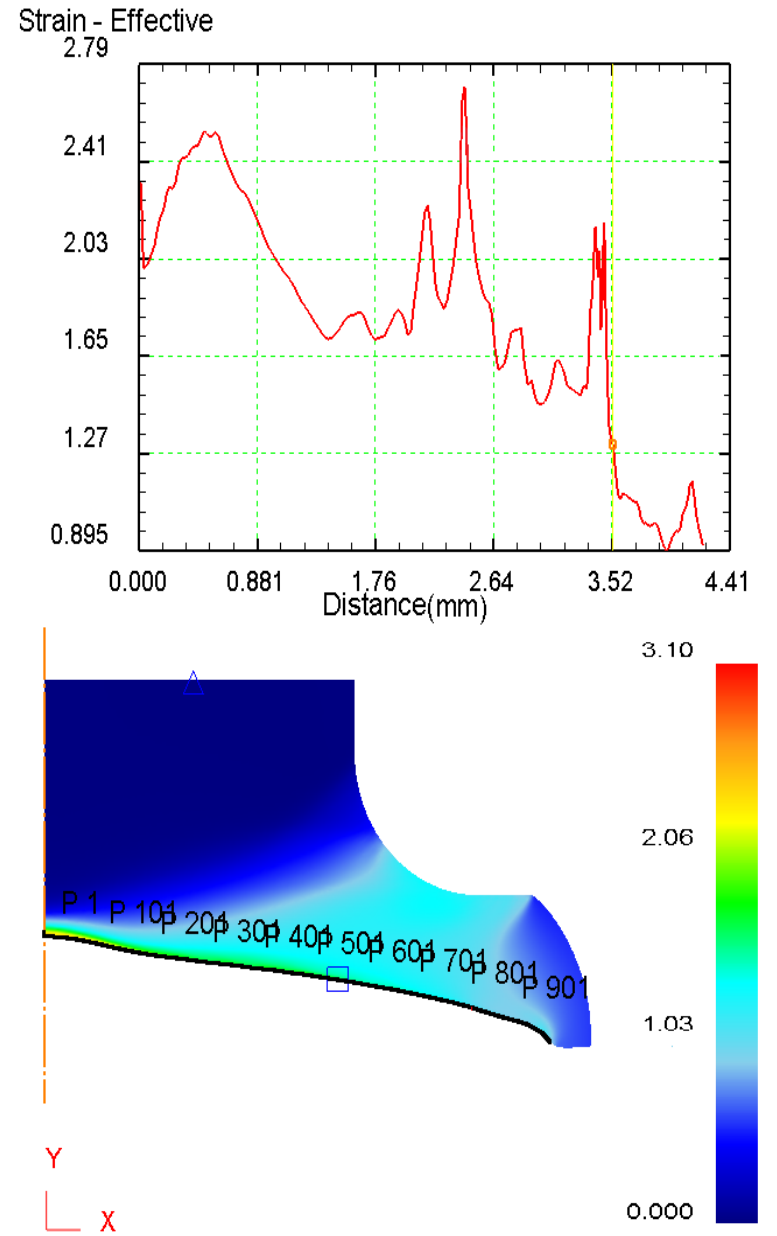

Fig. 9. Final stage of deformation, axial force equal to $21 \mathrm{kN}$.

In the last stage of deformation, the influence of micro-cutting disappear and mutual interaction within the contact zone is dominated by surface forces Fig. 9. The effect of the total disappearance of the impact of the roughness wave, is the results from limitations of the finite element method, in which the influence of friction is limited to the factor. In real conditions (experimental research), the impact of roughness as a result of large deformations does not disappear, but strongly interacts with the impacts of surface forces, causing for example plastic welds (adhesive welds), as a result of removing the oxide layers; as a result of micro-cutting and uncovering the deeper surface layers not yet oxidised and capable of forming adhesive bridges between the two metal surfaces, which has not been formulated by means of current computer methods. It should also be noted that the difference in the results of experimental research and the numerical experiment was $25 / 21 \mathrm{kN}$, corresponding to $16 \%$, and was caused by the fact that the results were often rounded off and simplified. However, the obtained results are very promising and indicate that it is necessary to further develop the presented method. 


\section{Conclusions}

Although it has been proven that roughness modelling is possible, it requires a different approach to the way we understand the processes of friction. The obtained results are very satisfying but indicate a need for the development of numerical methods adapted to issues related to friction. Through the application of available numerical methods and the presented mode of modulation, it is possible to estimate the effect of microcutting and surface force impact. Thanks to the development of numerical methods and the presented method, it will be possible to explain surface phenomena such as diffusion or adhesion, but the author believes that they will also prove useful in numerous fields of science and technology.

\section{References}

1. F. Sun, E. V. Giessen, L. Nicola, Wear, 296, 672680 (2012)

2. R. Buczkowski, M. Kleiber, J. Theor. App. MechPol., 4, 30 (1992)

3. X. Tan, Tribol. Int., 35, 385-393 (2002)

4. A. Brzoza, V. Pauk, J. Theor. App. Mech-Pol., 45, 2, 277-288, (2007)

5. S. Zhang, W. Wang, Z. Zhao, Tribol. Int., 79, 59-73 (2014)

6. A.V. Manoylov, J. Bryantm, H.P. Evans, Tribol. Int., 65, 248-258 (2013)

7. L.G. Wang, X.P. Sun, Y. Huang, J. Mater. Process. Tech., 187-188, 631-634 (2007) 